\title{
Il Platelet Activating Factor (PAF), marcatore di biocompatibilità, nella dialisi con membrane in poliacrilonitrile
}

\author{
C. Guastoni' ${ }^{1}$ C. Tetta ${ }^{2}$, N.A. Hoenich ${ }^{3}$, R. Gervasio ${ }^{2}$, L. Sereni², E. Tessore², \\ M.L. Wratten ${ }^{2}$, G. Civati ${ }^{1}$ \\ ${ }^{1}$ Unità Operativa di Nefrologia, Dialisi e Terapia del Trapianto Renale \\ Ospedale Niguarda Cà Granda, Milano \\ ${ }^{2}$ Centro di ricerca clinica BellCo SpA - Mirandola (MO) \\ ${ }^{3}$ Department of Medicine, University of Newcastle Upon Tyne - UK
}

I Platelet-Activating Factor (PAF) è uno dei mediatori cellulari dell'infiammazione e dell'anafilassi. La sua produzione, inizialmente attribuita solo alle piastrine $(1,2)$, è stata recentemente associata anche ai monociti, macrofagi, neutrofili, linfociti natural killer, cellule endoteliali, cellule renali glomerulari, e all'endotelio dei vasi coronarici in risposta ad appropriati stimoli (1-4).

La sintesi del PAF da parte dei macrofagi e delle cellule endoteliali, IgE mediata o stimolata da istamina e bradichinina, suggerisce un coinvolgimento del PAF nella patogenesi della reazione anafilattica.

Precedenti osservazioni, nostre e di altri Autori, hanno attribuito al PAF un ruolo nelle risposte cellulari legate al contatto sangue/membrana durante il trattamento emodialitico (5-10).

La sintesi ed il release di PAF è un evento transitorio, che interviene nelle primissime fasi dell'interazione tra il sangue e le membrane di dialisi attivanti il complemento.

In vitro una rilevante quantità di $\mathrm{PAF}$ è generata dopo l'incubazione di leucociti con il Cuprophan.

I meccanismi responsabili della produzione del PAF sono collegati all'attivazione complementare o derivano dalla sola interazione sangue/membrana, in- dipendentemente dalla presenza del complemento.

Studi recenti hanno riportato un'aumentata incidenza di reazioni anafilattiche durante il trattamento dialitico (1114). A differenza delle prime osservazioni che riguardavano prevalentemente pazienti sottoposti ad emodialisi con Cuprophan $(15,16)$ le osservazioni più recenti hanno posto l'attenzione sul ruolo dell'uso combinato degli ACE inibitori delle membrane in AN 69 (14). Si è ipotizzato che le cariche negative poste sulla superficie dell'AN69 possano indurre l'attivazione del fattore di Hagemann e la conversione della precallicreina in callicreina che conduce alla generazione di bradichinina (17). La bradichinina possiede diverse attività biologiche, quali quella di produrre ipotensione e broncospasmo attraverso vasodilatazione e brococostrizione (18). Numerose reazioni correlate all'anafilassi sono dovute alla produzione di mediatori indotti dalla bradichinina.

Quest'ultima è in grado di stimolare la produzione di prostaglandine, istamina e PAF (19). Poiché il PAF risulta biologicamente dalle 100 alle 1000 volte più attivo dell'istamina nel produrre vasodilatazione $(1,2)$, lo scopo del presente studio è stato di valutare, in vitro ed in vivo, se avviene sintesi e release di PAF durante dialisi con membrane in Polia- crilonitrile AN 69. Per indagare il possibile ruolo delle cariche negative poste sulla superficie della membrana abbiamo studiato la capacità di indurre la sintesi del PAF con una membrana in poliacrilonitrile nella quale le cariche di superficie sono state modificate e con la quale recentemente è stata provata la mancata produzione di bradichinina (20). Infine abbiamo studiato in vitro i meccanismi implicati nella sintesi del PAF utilizzando sia il Cuprophan che il Poliacrilonitrile.

\section{Pazienti, materiali e metodi}

Abbiamo esaminato 11 pazienti età media 65.1 \pm 11.1 anni; (54-76 anni; 7 maschi e 4 femmine).

Tutti eseguivano emodialisi cronica trisettimanale da una media di 87.9 mesi (11-94 mesi).

In tutti venivano usate membrane sintetiche con superficie $1.6 \mathrm{~m}^{2}$. Il bagno dialisi aveva la seguente composizione: $\mathrm{Na}^{+} 140$, $\mathrm{K}^{+} 2, \mathrm{Ca}^{++} 1.5$, Acetato ${ }^{-} 40, \mathrm{Cl}^{-} 100$, $\mathrm{Mg}^{++} 0.75 \mathrm{mMol} / \mathrm{L}$ e glucosio $1 \mathrm{~g} / \mathrm{L}$. La durata del trattamento era di 4 ore. Nessun paziente era trattato con ACE inibitore e/o aveva una storia clinica di malattia autoimmune o di anafilassi. 
I monitor utilizzati nello studio erano tutti con controllo automatico dell'ultrafiltrazione.

I dializzatori utilizzati per la sperimentazione erano il Filtral 16 (Hospal) in Poliacrilonitrile AN 69 ed il PA 1630 (Bellco SpA, Mirandola, Italia) in membrana SPAN.

La superficie dei dializzatori era di $1.6 \mathrm{~m}^{2}$.

\section{Protocollo di studio}

Le membrane testate sono state utilizzate in ogni paziente per due settimane secondo lo schema sotto esposto.

La sperimentazione è stata condotta nella seconda settimana di trattamento.

I settimana $^{\text {AN } 69}$ II settimana

valutazione in vivo

$\frac{\text { I settimana }_{\text {SPAN }}^{\text {II settimana }}}{\text { valutazione in vivo }}$

\section{Studio clinico}

Tutti i pazienti sono stati trattati con AN 69 di $1.6 \mathrm{~m}^{2}$ di superficie (Hospal) per 3 mesi prima dell'inizio dello studio.

I pazienti sono stati assegnati in modo randomizzato all'AN 69 (5 pazienti) allo SPAN (6 pazienti) per due settimane. La sperimentazione è stata condotta nella seconda settimana di utilizzo. Dopo aver completato la seconda settimana tutti i pazienti sono passati al trattamento con la seconda membrana per le successive due settimane.

La valutazione della biocompatibilità e delle performance dialitiche è stata eseguita nella seconda seduta della seconda settimana di studio.

\section{Biocompatibilità}

I leucociti e le piastrine sono stati determinati su sangue prelevato dalla via arteriosa.

Le frazioni del complemento C3a e C5b-C9 sono state determinate su prelievi eseguiti dalla linea venosa in provette contenenti EDTA mantenute in ghiaccio e successivamente centrifugate a $2000 \mathrm{~g}$ per 10 minuti a $4^{\circ} \mathrm{C}$.

Una soluzione stabilizzante il complemento (Quindel, San Diego, USA) è stata aggiunta al plasma $1: 1 \mathrm{v} / \mathrm{v}$ e que- sto è stato conservato a $-70^{\circ} \mathrm{C}$. Le concentrazioni di $\mathrm{C} 3 \mathrm{a}$ e $\mathrm{C} 5 \mathrm{~b}-\mathrm{C} 9$ sono state misurate con metodo EIA (Quindel, San Diego, USA).

\section{Clearance}

Le clearance di urea, creatinina e fosfato sono state determinate in periodi di 15 minuti a 30 ', 120 ' e 180 ' dopo l'inizio del trattamento dialitico secondo la seguente formula: $\mathrm{D} \times \mathrm{V} / \mathrm{P}$; dove $\mathrm{D}=$ concentrazione del soluto nel dialisato raccolto in uscita dal filtro, $\mathrm{V}=$ volume del dialisato raccolto per $15^{\prime}, \mathrm{P}=$ concentrazione del soluto nel plasma all'ingresso del filtro a metà del periodo di clearance.

Le concentrazioni di urea, creatinina e fosfato sono state determinate con Syncon Clinical Analyzer (Beckman Instruments Inc. USA).

La Beta 2 - microglobulina è stata determinata nel plasma pre e post-trattamento e nel dialisato totale effluente con metodo immunonefelometrico (Eiken Chemical, Tokio, Japan).

\section{Risonanza magnetica nucleare delle membrane (NMR)}

Membrane SPAN e AN 69 sono state prelevate dai dializzatori e disciolte in dimetilsulfossido $(4 \% \mathrm{v} / \mathrm{v})$. Lo spettro protonico in NMR è stato ottenuto con uno spettrometro NMR ad alta risoluzione $(250 \mathrm{MHz})$ con una testa a doppio sensore di $5 \mathrm{~mm}$ a $30^{\circ} \mathrm{C}$.

\section{Studio in vitro}

Il sangue intero fresco eparinato o gli eritrociti lavati sono stati fatti ricircolare in circuito chiuso con flusso di $300 \mathrm{ml} / \mathrm{min}$ in dialisi con bagno riscaldato a $37^{\circ} \mathrm{C}$ contenente tampone acetato. Si sono utilizzati tre tipi di membrana dialitica: AN69, SPAN-S e Cuprophan. Gli eritrociti lavati sono stati preparati dopo l'eliminazione del plasma mediante centrifugazione $(1200 \mathrm{~g}$ per $20 \mathrm{~min}$ ) e due lavaggi in una soluzione tampone sterile a pH 7.2 (25). Gli eritrociti sono stati riportati all'ematocrito originale con l'aggiunta di una soluzione Tris-Tyrode e di una soluzione contenente albumina bovina $0.25 \%$.

Il numero dei leucociti e delle piastrine è stato dosato con counter coulter.

Il PAF è stato dosato dopo estrazione lipidica sul sangue e sulle piastrine.

Al fine di dosare la concentrazione di
PAF legato alle membrane dialitiche il comparto ematico e quello del dialisato sono stati perfusi con $100 \mathrm{ml} \mathrm{di} \mathrm{me-}$ tanolo assoluto acidificato con acido acetico e si sono mantenuti in agitatore per un'ora. Successivamente il metanolo è stato aspirato, centrifugato a $2000 \mathrm{~g}$ per $15 \mathrm{~min}$ e stratificato su una soluzione cloroformio-acqua 1/0.9 vol/vol. Il cloroformio è stato aspirato e fatto evaporare. Il PAF è stato purificato e dosato secondo i metodi descritti di seguito.

\section{Estrazione, purificazione e dosaggio del PAF}

Il PAF è stato estratto e purificato dal plasma ottenuto da $5 \mathrm{ml}$ di sangue (dalla via arteriosa al tempo 0 e dalla via venosa ai tempi $2,5,15 \mathrm{~min}$ ) prelevato in soluzione acido citrico-destrosio in 5 pazienti. Le tecniche di estrazione, purificazione e di dosaggio sono quelle precedentemente descritte (21).

\section{Analisi statistica}

I dati sono stati analizzati usando l'analisi della varianza a misurazioni ripetute quando necessarie. L'analisi statistica è stata eseguita usando il t test per dati appaiati e non appaiati.

\section{Risultati}

\section{Sintesi e release del PAF durante emodialisi}

Nelle primissime fasi del trattamento con AN 69 in tutti i 5 pazienti si è osservato un aumento dei livelli di PAF libero nel plasma che sulla superficie delle piastrine (rispettivamente $4.5 \pm 2.7 \mathrm{ng} / \mathrm{ml}$ e $4.1 \pm 1.2 \mathrm{ng} / \mathrm{ml}$ ). Al contrario, nessuna variazione rispetto al valore iniziale è stata osservata durante le dialisi con la membrana SPAN (Fig. 1A e B) In tutti i pazienti la concentrazione del PAF ha raggiunto il massimo valore a 2 min dall'inizio e si è ridotta rapidamente $(1.1 \pm 0.8 \mathrm{ng} / \mathrm{ml})$ a $5 \mathrm{~min}$. A $15 \mathrm{~min}$ dall'inizio della procedura non vi era presenza di PAF.

La produzione del PAF non era correlata all'attivazione complementare, infatti, come è mostrato in Tabella I, il comportamento dei leucociti e delle piastrine non mostrava differenze tra le due membrane. A 15 min dall'inizio si è osservata una riduzione del numero dei 


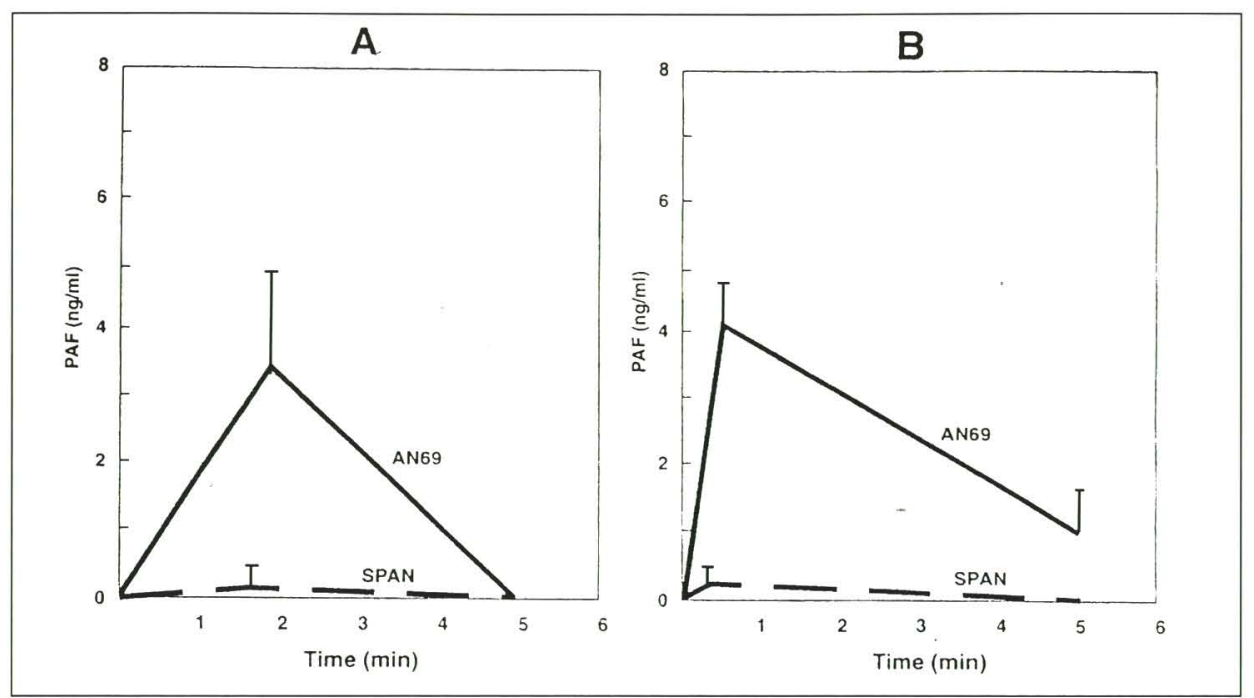

Fig. 1 - Concentrazione del PAF libero nel plasma $(A)$ ed associato alle piastrine $(B)$, durante emodialisi in vivo con AN 69 e con SPAN.

TAB. I - COMPORTAMENTO DEI LEUCOCITI, DELLE PIASTRINE E CONCENTRAZIONE DELLE FRAZIONI COMPLEMENTARI DURANTE DIALISI $I N V I V O$ CON AN 69 E CON SPAN

\begin{tabular}{lccccc}
\hline & & $\begin{array}{c}\text { Leucociti } \\
\left(/ \mathrm{mm}^{3}\right)\end{array}$ & $\begin{array}{c}\text { Piastrine } \\
\left(\times 10^{3} / \mathrm{mm}^{3}\right)\end{array}$ & $\begin{array}{c}\text { C3a } \\
(\mathrm{ng} / \mathrm{ml})\end{array}$ & $\begin{array}{c}\text { C5b-C9 } \\
(\mathrm{ng} / \mathrm{ml})\end{array}$ \\
\hline \multirow{2}{*}{0} & AN 69 & $4950 \pm 574$ & $145 \pm 45$ & $255 \pm 140$ & $27 \pm 17$ \\
& SPAN & $5275 \pm 788$ & $163 \pm 51$ & $216 \pm 81$ & $47 \pm 46$ \\
& & & & & \\
5 & AN 69 & $4851 \pm 572$ & $141 \pm 42$ & $408 \pm 383$ & $88 \pm 71$ \\
& SPAN & $5171 \pm 791$ & $160 \pm 48$ & $412 \pm 312$ & $14 \pm 10$ \\
& & & & & \\
15 & AN 69 & $4200 \pm 812$ & $142 \pm 48$ & $310 \pm 378$ & $167 \pm 107$ \\
& SPAN & $4625 \pm 869$ & $151 \pm 42$ & $333 \pm 188$ & $152 \pm 120$ \\
30 & AN 69 & $4975 \pm 942$ & $144 \pm 52$ & $216 \pm 249$ & $162 \pm 110$ \\
& SPAN & $5175 \pm 960$ & $156 \pm 44$ & $293 \pm 151$ & $197 \pm 108$ \\
50 & AN 69 & $4925 \pm 1117$ & $150 \pm 53$ & $207 \pm 191$ & $160 \pm 150$ \\
& SPAN & $4850 \pm 1100$ & $157 \pm 42$ & $271 \pm 110$ & $141 \pm 79$ \\
\hline
\end{tabular}

leucociti con entrambe le membrane (riduzione del $18.4 \%$ rispetto al valore basale) senza differenze statisticamente significative $(p>0.05)$.

Le piastrine non hanno mostrato riduzioni durante il trattamento senza variazioni significative tra le due membrane. I livelli di C3a desArg e del complesso C5b-C9 non hanno mostrato differenze tra SPAN e AN 69. La concentrazione di $\mathrm{C} 3 \mathrm{a}$ des Arg ha raggiunto il valore massimo (408.28 e $412.85 \mathrm{ng} / \mathrm{ml}$ rispettivamente con AN 69 e con SPAN) 5 min dopo l'inizio della circolazione extracorporea, per ridursi in seguito con entrambe le membrane. La concentrazione del complesso $\mathrm{C} 5 \mathrm{~b}-\mathrm{C} 9$ ha raggiunto il suo massimo valore a $15 \mathrm{~min}$ con entrambe le membrane (197.1 e $162.22 \mathrm{ng} / \mathrm{ml}$ rispettivamente con AN 69 e SPAN).

La Figura 2 riassume i risultati delle clearance di urea, creatinina e fosfato. I valori non evidenziano alcuna differenza tra le due membrane.

Sia lo SPAN che l'AN-69 si sono dimostrati efficaci nel ridurre i livelli plasmatici di Beta 2 - microgloblulina (Tab. II), tuttavia la rimozione per dialisi è risultata significativamente maggiore con lo SPAN rispetto all'AN 69, (Fig. 3).

\section{Risultati in vitro}

La Figura 4 mostra lo spettro NMR delle due membrane testate. La più evidente differenza tra lo SPAN e l'AN 69 è la notevole riduzione dei gruppi sulfonici della superficie dello SPAN rispetto all'AN 69 (0.4\% vs $3.2 \%)$.

La Figura 5 mostra i livelli di PAF riscontrati nel sangue intero dopo 2,5 e 15 min di circolazione extracorporea.

TAB. II - CONCENTRAZIONE PLASMATICA DELLA BETA 2-MICROGLOBULINA PRE E POST-TRATTAMENTO E VARIAZIONE (DELTA \%) DELLA CONCENTRAZIONE RISPETTO ALL'INIZIO DEL TRATTAMENTO. I valori post non sono stati corretti per la variazione dell'ematocrito

\begin{tabular}{|c|c|c|c|c|c|c|}
\hline \multirow[t]{2}{*}{ Beta 2-micro } & SPAN & 1.6 & & $5 \mathrm{pz}$ & & \\
\hline & PRE & POST & Delta \% & QB & Delta peso & Peso finale \\
\hline MEDIA & 44.3 & 25.6 & 41.7 & 267 & 2,3 & 52,8 \\
\hline dev st & 11.6 & 5.5 & 5.2 & 26 & 0,8 & 8,1 \\
\hline \multirow[t]{2}{*}{ Beta 2-micro } & FILTRAL & 1,6 & & $5 \mathrm{pz}$ & & \\
\hline & PRE & POST & Delta \% & QB & Delta peso & Peso finale \\
\hline MEDIA & 39.1 & 25.4 & 35.4 & 278.3 & 2,3 & 52,6 \\
\hline dev st & 6.1 & 6.2 & 7.0 & 24.8 & 0,7 & 8,0 \\
\hline
\end{tabular}




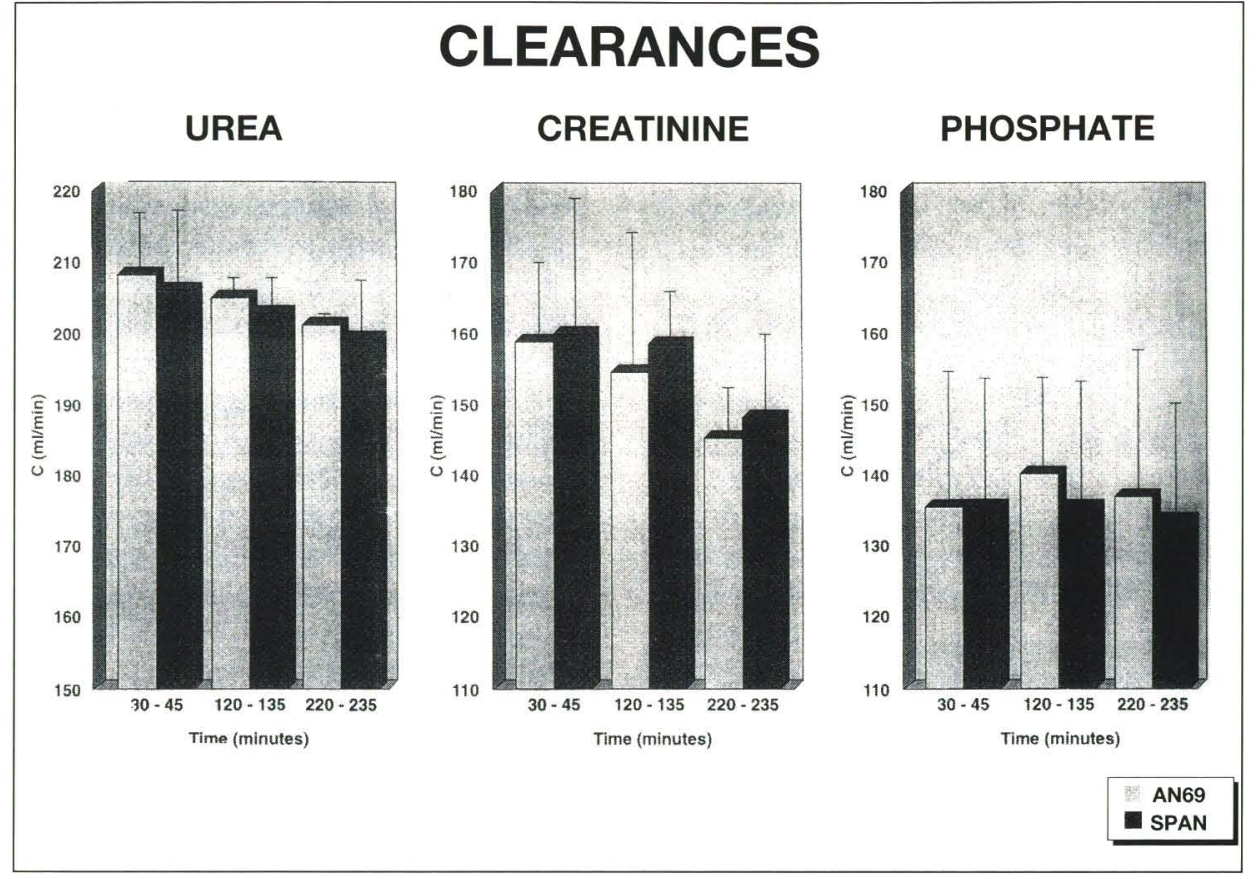

Fig. 2 - Clearance di urea (A), creatinina (B), e fosfato $(C)$ durante emodialisi in vivo con AN 69 (colonne scure) e con SPAN (colonne chiare).
Una produzione $\mathrm{d}$ PAF si è resa evidente con entrambe le membrane, tuttavia la quantità di PAF generato con l'AN 69 era maggiore di quella prodotta con la membrana SPAN. Inoltre lo SPAN agisce adsorbendo una maggiore quota di PAF rispetto all'AN 69. Ciò è dimostrato dalle maggiori concentrazioni di PAF rilevate, sia sulla superficie interna $(p<0.005)$, che su quella esterna ( $p<0.0001)$, dopo estrazione con metanolo alla fine di $20 \mathrm{~min}$ di circolazione in circuito chiuso (Fig. 6).

La membrana AN 69, ed in minore misura anche lo SPAN, è in grado di innescare la produzione di PAF solo in presenza di sangue intero. Viceversa non si è osservata generazione di PAF utilizzando il sangue lavato. In quest'ultimo il numero dei leucociti e delle piastrine era rispettivamente $3.2 \pm 0.5\left(\times 10^{9} / \mathrm{L}\right)$ e di $10.2 \pm 0.3\left(\times 10^{9} / \mathrm{L}\right)$.

$\mathrm{Al}$ contrario il Cuprophan è in grado di innescare la generazione di PAF sia su sangue intero, che lavato (Tab. III).

\section{B-2 microglobulin removal (mg)}

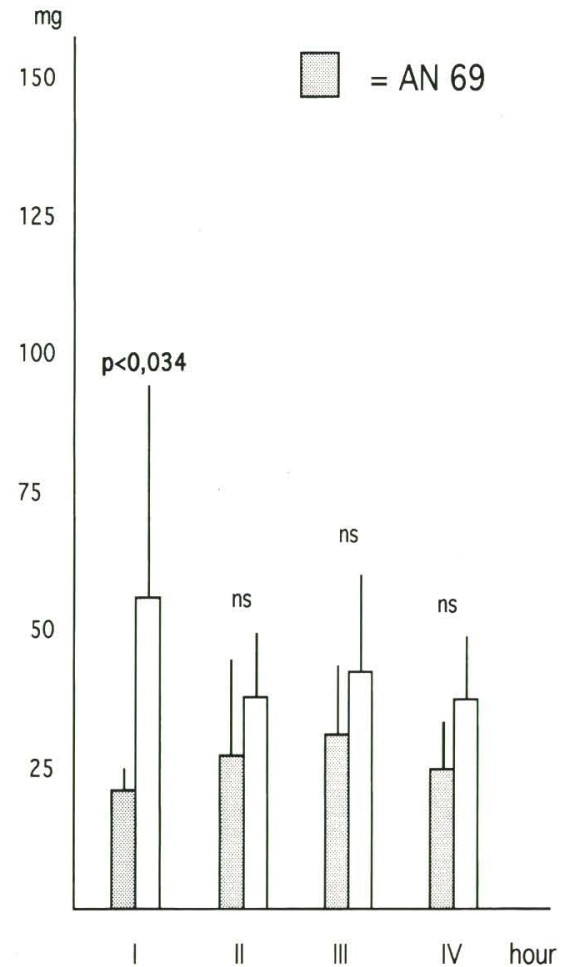

Fig. 3 - Rimozione della Beta 2-microglobulina durante dialisi con AN 69 e SPAN. Sono state misurate ogni ora le concentrazioni sul dialisato effluente. La rimozione totale è stata ottenuta dalla somma delle misurazioni orarie.

\section{NUCLEAR MAGNETIC RESONANCE AN69 VS SPAN}

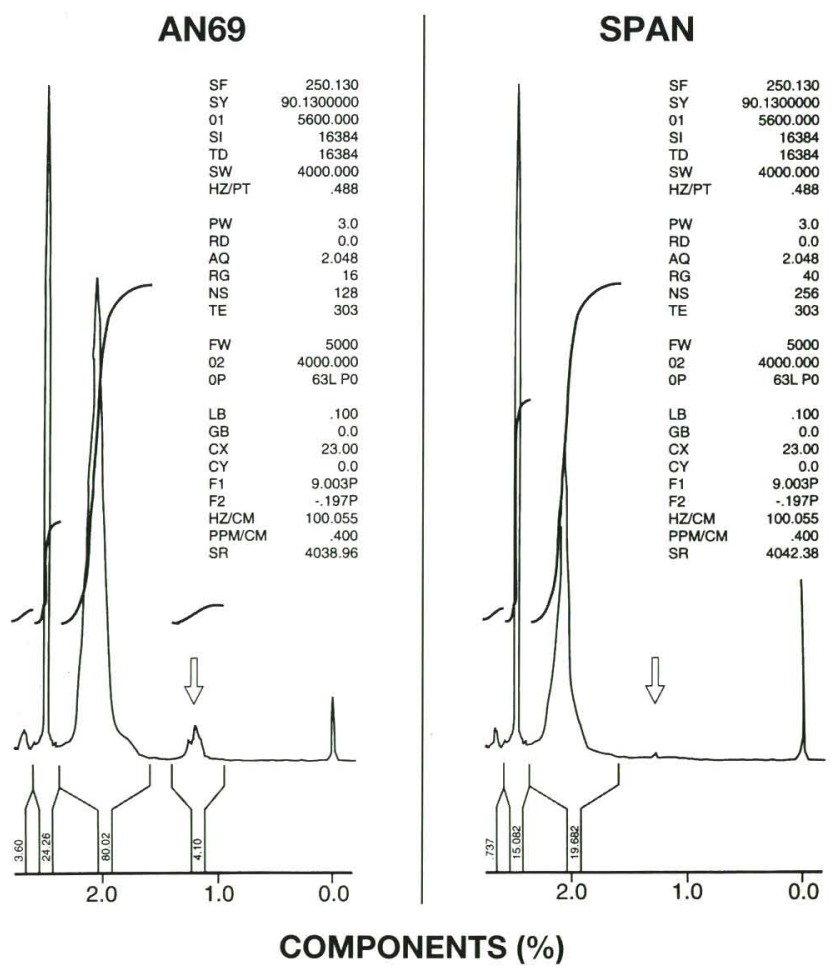

\begin{tabular}{lr}
\hline Acrylonitrile & 95.6 \\
Comonomer & 4.0 \\
Sulfonic acid group & 0.4
\end{tabular}

Fig. 4 - Studio spettroscopico in NMR dell' AN 69 e dello SPAN. 


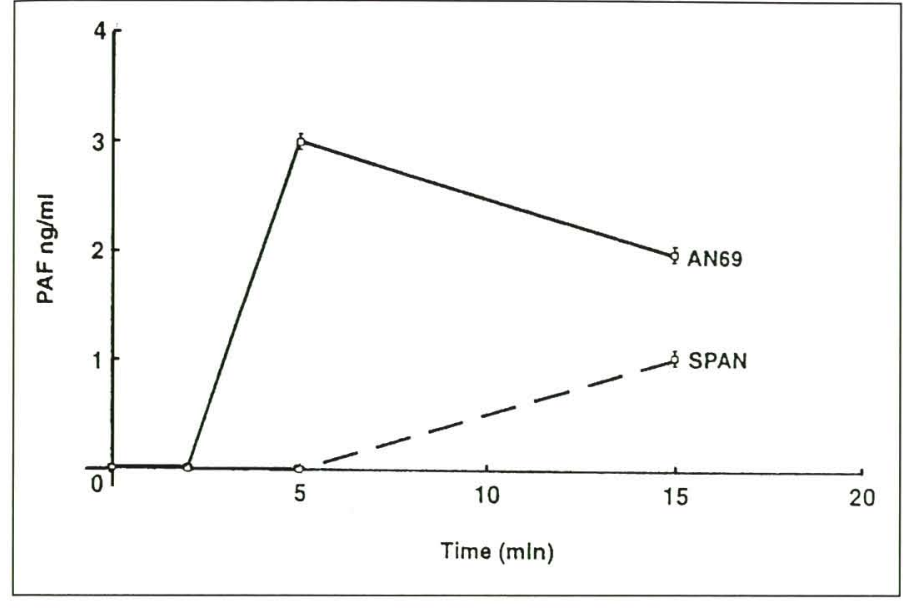

Fig. 5 - Concentrazione del PAF durante dialisi in vitro con sangue intero con AN 69 e con SPAN.

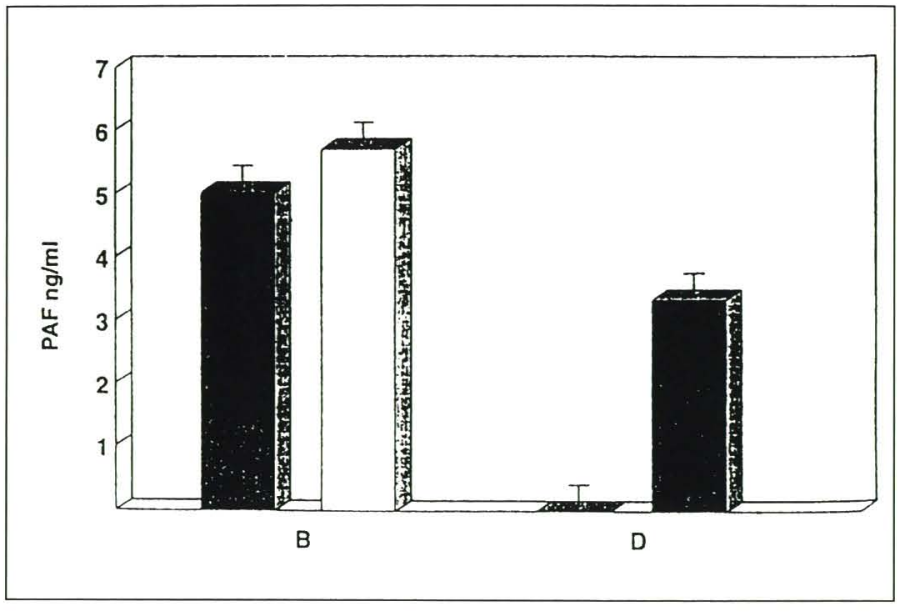

Fig. 6 - Concentrazione del PAF estratto con metanolo dal comparto ematico (B) e da quello del dialisato $(D)$ dopo dialisi in vitro con AN 69 (colonne chiare) e con SPAN (colonne scure).

\section{Discussione}

Nella parte clinica del nostro studio abbiamo paragonato le performance dialitiche e la produzione del PAF, come indice di biocompatibilità, di due tipi di membrana in Poliacrilonitrile. Per quanto il polimero di base costituente le due membrane sia lo stesso, il processo di produzione ne rende diversa la composizione finale.

La differente composizione finale non ha comportato differenze in termini di performance depurative.

Viceversa la valutazione della biocompatibilità evidenzia importanti differenze.

La membrana AN 69 induce sintesi e release di PAF durante la dialisi in vivo. Tale fenomeno risulta essere presente nelle primissime fasi del trattamento, con un massimo a 2 minuti, non essendo più determinabile dopo 5 minuti dall'inizio del trattamento.

Precedenti studi riguardanti la sintesi del PAF hanno evidenziato la responsabilità di due possibili meccanismi: il primo plasma dipendente e mediato dallo stimolo del C5a-C5a des Arg, il secondo, indipendente dal complemento e legato all'interazione tra sangue e membrana dialitica (22).

Recentemente l'osservazione che le cariche negative poste sulla superficie della membrana possono attivare la produzione di bradichinina, ha fornito la possibile prova di un terzo meccani- smo di attivazione e release del PAF che risulta mediato dal plasma, ma indipendente dalla presenza del complemento.

È noto che la bradichinina possiede sulle cellule endoteliali dei recettori specifici (Beta-2) (18) la cui stimolazione induce una sintesi di PAF dose dipendente con un massimo a 5 minuti (23).

Il presente lavoro suggerisce che la produzione di PAF con la membrana AN 69 sia innescata dall'attivazione di una via biosintetica dipendente dalla presenza del plasma.

Inoltre la cinetica del release del PAF si è dimostrata più rapida di quella osservata con le colture di cellule endoteliali (19). Ciò potrebbe essere spiegato con un coinvolgimento di altre cellule circolanti in grado di produrre PAF come $\mathrm{i}$ leucociti e le piastrine.

Nelle sperimentazioni in vitro il PAF veniva prodotto solo in presenza di sangue integro, e non con sangue lavato privato della componente plasmatica.

In accordo con studi precedenti (24) la produzione di PAF è stata indotta in dialisi in vitro a circuito chiuso con una membrana cellulosica, sia utilizzando sangue intero, che eritrociti lavati.

Questo studio non è stato in grado di giungere ad una dimostrazione conclusiva che la bradichinina sia responsabile della sintesi e del release di PAF con AN 69. Tuttavia questa ipotesi è fortemente suggestiva in quanto la riduzione delle cariche negative sulla superficie dello SPAN, dedotta dalla quasi totale assenza di gruppi sulfonici, impedisce la sintesi ed il release di PAF in emodialisi in analogia con la riduzione della produzione di bradichinina da altri osservata (20).

Nella pratica clinica va considerato che esiste una variabilità individuale nei livelli plasmatici di bradichinina (Van der Niepen et al 1995). La complessità della reazione anafilattica non è solo dovuta a questa variabilità individuale, ma anche alla sensibilità dell'organismo all'attività indotta dai mediatori biologici ed alla loro concentrazione nei tessuti. Tutti questi fattori possono ben rendere ragione della mancanza di una correlazione diretta tra i livelli plasmatici degli stessi mediatori e l'espressione clinica della loro attività.

In termini di biocompatibilità il Poliacrilonitrile AN 69 induce i più elevati livelli di bradichinina plasmatica (11, 12, 14).

Analogamente alla bradichinina, anche la dimostrazione della generazione di PAF con l'AN 69 non prova in maniera definitiva, con rapporto causa-effetto, un coinvolgimento di questo mediatore cellulare nella genesi delle reazioni anafilattiche dei pazienti sottoposti ad emodialisi con AN 69 ed in terapia con ACE inibitori. Pur tuttavia questo risultato sta a dimostrare che l'interazione tra il sangue e le cariche negative poste sulla superficie dell'AN 69 può innescare non soltanto la produzione di mediatori plasmatici (bradichinina), ma anche di mediatori cellulari (PAF). La loro azione comune può essere implicata nei meccanismi che sono alla base delle reazioni di bioincompatibilità $(25,26)$. 
TAB. III - RELEASE DI PAF IN DIALISI IN VITRO CON DIFFERENTI MEMBRANE DIALITICHE SU SANGUE INTERO E SU EMAZIE LAVATA

\begin{tabular}{llcc}
\hline & & PAF & \\
min & membrana & sangue intero & emazie \\
\hline 0 & AN 69 & $0.2 \pm 0.12$ & $0.4 \pm 0.20$ \\
& SPAN & $0.11 \pm 0.2$ & $0.3 \pm 0.10$ \\
& Cuprophan & $0.11 \pm 0.2$ & $0.11 \pm 0.2$ \\
2 & AN 69 & $4.40 \pm 2.50$ & $0.4 \pm 0.1$ \\
& SPAN & $0.30 \pm 0.10^{*}$ & 0 \\
& Cuprophan & $8.5 \pm 2.4$ & $10.5 \pm 4.40$ \\
\multirow{2}{*}{15} & AN 69 & $1.13 \pm 0.50$ & $0.6 \pm 1.10$ \\
& SPAN & 0 & 0 \\
& Cuprophan & $4.5 \pm 2.4$ & $8.9 \pm 2.6$ \\
\hline$*=\mathrm{p}<0.05$ & & & \\
& & &
\end{tabular}

Può essere argomentato che le concentrazioni di PAF possano venire sottostimate a causa del suo legame con le superfici cellulari, per effetto delle antiproteasi e per l'azione di inattivazione da parte dell'enzima specifico Acetilidrolasi, presente nel plasma ed in molte cellule dell'organismo.

Una carente attività acetilidrolasica può assumere rilevanza clinica in condizioni particolari nelle quali si ha aumento del PAF nei fluidi biologici, come avviene nel plasma nel caso di riacutizzazioni del Lupus Eritematoso Sistemico (21).

Non è noto se nei pazienti sottoposti ad emodialisi i meccanismi che regolano la sintesi e la degradazione del PAF siano alterati.

Infatti i livelli plasmatici potrebbero essere il frutto di una esagerata produzione, in grado di superare la capacità di degradazione, o della presenza di un meccanismo inibente la via di degradazione del PAF. In più la elevata capacità adsorbitiva dimostrata al Poliacrilonitrile può portare ad una sottostima della quantità di PAF prodotta in dialisi.

In tal senso ulteriori studi sono necessari per chiarire se la membrana SPAN induca effettivamente una minore produzione di PAF rispetto al poliacrilonirile AN 69, oppure se le minori concentrazioni di PAF osservate con lo SPAN siano il risultato di una sua maggiore capacità adsorbitiva.

\section{Riassunto}

È noto che il platelet-activating factor (PAF) è uno dei mediatori solubili dell'anafilassi.

Abbiamo studiato, in vivo ed in vitro, $\mathrm{i}$ meccanismi e la cinetica di produzione del PAF con membrana dialitica in poliacrilonitrile AN 69 e con un suo derivato nel quale la componente in Na-metallilsulfonato è stata parzialmente sostituita con il metacrilato, con minori cariche elettriche negative (SPAN).

Il PAF si è reso dosabile un minuto dopo l'inizio del trattamento, sia su sangue intero $(44.5 \pm 2.7 \mathrm{ng} / \mathrm{ml})$, che sulla superficie delle piastrine $(4.1 \pm 1.2$ $\mathrm{ng} / \mathrm{ml}$ ) solo durante il trattamento con AN 69, mentre era indosabile nelle dialisi con lo SPAN. I leucociti, le piastrine e le frazioni del complemento non hanno mostrato differenze significative tra le due membrane.

La modifica della struttura del Poliacrilonitrile dello SPAN non ne ha alterato le performance depurative, come è dimostrato dall'assenza di differenze significative tra le clearance di piccole molecole e l'estrazione della Beta 2 microglobulina ottenute con le due membrane.

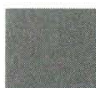

\section{BIBLIOGRAFIA}

1. Snyder F. Platelet-activating factor and related acetylated lipids as potent biological acting cellular mediators. Am J Physiol 1990; 259: 697.

2. Camussi G. Tetta C, Baglioni C. The role of platelet-activating factor in inflammation. Clin Immun Immunopath 1990; 57: 331.

3. Schtondorff D, Neuwirth R. Platelet-activating factor and the kidney. Am J Physiol 1986; 251: F1.

4. Montrucchio G, Bergerone S, Bussolino F et al. Streptokinase induces intravascular release of platelet-activating factor and prostacyclin in patients with actue myocardial infarction and stimulates its synthesis by cultured endothelial cells. Circulation 1993; 88: 1476.

5. Mandrenas J, Pintos Morell G, Bratolome E et al. PAF-acether (platelet-activating factor) and biocomptibility of hemodialysis membranes. Kidney Int 1987; 32: 614.

6. Gonzalez-Lopez A, Fernandez-Gallardo S, Caramela C, Inarrea P, Hernando L, Sanchez Crespo M. Presence of PAF-acether in blood during hemodialysis (HD) and its relation with dialysis leukopenia. Kidney Int 1985; 28: 587.

7. Cristol JP, Canaud B, Damon M, Chavis C, Arnoux B, Mion C. Platelet-activating factor production during hemodialysis: effect of $\mathrm{BN}$ 52021. Prostaglandins-Leukot-Essent. Fatty Acids 1992; 45: 37.

8. Sakaguchi K, Morimoto S, Chen J-H, Nakamoto Y, Ogihara T. Increase in circulating level of platelet-activating factor lag behind transient neutropemia during hemodialysis with cupraphan membrane. Nephron 1991; 59: 445.

9. Tetta C, David S, Biancone L et al. Role of platelet-activating factor in hemodialysis. Kidney Int 1993; 43: 514. 
10. Iarrou C, Afenkatis N, Antonopoulou $\mathrm{S}$ et al. The production of platelet-activating factor (PAF) during hemodialysis with cuprophan membrane. Does the calcium concentration in the dialysate play any role on it? Int J Artif Organs 1995; 18: 355-61.

11. Tielemans C, Madhoun P, Lenaers $\mathrm{M}$ et al. Anaphylactoid reactions during hemodialysis on AN-69 membranes in patients receiving ACE inhibitors. Kidney Int 1990; 38: 982 .

12. Alvarez-Lara MA, Martin-Malo A, Espinoza M, Castillo D, Alfama P. ACE inhibitors and anaphylactoid reactions to high-flux membrane dialysis (Letter). Lancet 1991; 337: 370 .

13. Jadoul M, Stuyven J, Stragier A, Van Ypersele de Strihou C. Angiotensin-converting-enzyme inhibitors and anaphylactoid reactions to high-flux membrane dialysis (Letter). Lancet 1991; 337: 112.

14. Parnes El, Shapiro WB. Anaphylactoid reactions in hemodialysis patients treated with the AN69 dialyzer. Kidney Int 1991; 40: $114 \mathrm{~S}$.

15. Popli S, Ing TS, Daugirdas JT et al. Severe reactions to cuprophan capillary dialyzers. Artif Organs 1982; 6: 312.

16. Hakim RM, Breillatt J, Lazarus JM, Port FK. Complement activation and hypersensitivity reactions to dialysis membrane. N Engl J Med 1984; $311: 878$.

17. Verresen L, Fink E, Lemke HD et al. Bradykinin is a mediator of anaphylactoid reactions during hemodialysis with AN69 membranes. Kidney Int 1994; 45: 1497.

18. Bhoola KD, Figueroa CD, Worthy K. Bioregulation of kinase kallikreins, kininogens and kininases. Pharmacol Rev 1992; 44: 1.

19. Mc Intyre TM, Zimmerman GA, Satoh K, Prescott SM. Cultured endothelial cells synthesize both platelet-activating factor and pro- stacyclin in response to histamine, bradykinin and adenosine triphosphate. J Clin Invest 1985; 76: 271.

20. Mannstadt M, Tonam M, Fink E, et al. No generation of bradykinin with a new PAN membrane (SPAN) in dialysis patients treated with ACE inhibitors. EDTA. Athens Abstracts Book 1995; 248.

21. Tetta C, Bussolino F, Modena $\mathrm{V}$ et al. Release of platelet-activating factor in Systemic Lupus Erythematosus. Int Arch Allergy Appl Immunol 1990; 91: 241.

22. Camussi G, Segoloni C, Rotunno M, Vercellone A. Leukocyte derived mediators and biocampatibility. Nephrol Dial Transplant 1991; 2: 24 .

23. Mathews JNS, Hoenich NA. Statistical aspects of the design and analysis of studies to compare hemodialysis membranes. Nephrol Dial Transplant 1994; 9 (suppl 2): 176.

24. Tetta C, Segoloni G, Pacitti A, Camussi C, Vercellone A. The production of platelet-activating factor in hemodialysis. Int $\mathbf{J}$ Artif Organs 1989; $12: 766$.

25. Ronco C, Tetta C. Lupi A et al. Removal of platelet-activating factor in experimental continuous arteriovenous hemofiltration. Crit Care Med 1995; 23: 99.

26. Van der Niepen P, Sennesael JJ, Verbeelen DL. Kinin kinetics during different dialysis protocols with AN69 dialyser in ACEI. Treatet patients. Nephrol Dial Transplant 1995; 10: 1689. 